\title{
MECHANICAL STRENGTH OF STRIPPED OPTICAL FIBER
}

\author{
R. El Abdi1, a, R. Leite Pinto ${ }^{1,2}$, P. Lallinec ${ }^{2}$ and M. Poulain ${ }^{3}$ \\ ${ }^{1}$ Université de Rennes- CNRS, Institut de Physique de Rennes. UMR 6251 \\ F-35000 Rennes, France \\ ${ }^{2}$ Entreprise Acome- Usines de Mortain, 50140 Mortain, France \\ ${ }^{3}$ Institut des Sciences Chimiques de Rennes-UR1, UMR CNRS 6226 \\ 35042 Rennes Cedex, France \\ arelabdi@univ-rennes1.fr
}

\begin{abstract}
A novel removal technique of silica optical fiber coating with chemical stripping gel was used. We observed that the fiber strength decreases from 5.48 to $4.96 \mathrm{GPa}$ in the case of tensile test and from 0.06 to $0.05 \mathrm{GPa}$ in the case of bending test when the coating was removed from the cladding surface. But the stress corrosion parameter varies little considering the measurement accuracies.
\end{abstract}

Keywords: Optical Fiber; Coating; Stripping Gel; Tensile Test; Bending Test; Stress Corrosion Parameter.

\section{Introduction}

The commercial single-mode fibers are typically composed of silica glass for the core and cladding and acrylate protective coating. Sakaguchi and Hibino [1] were interested by the fatigue in the lowstrength of silica optical fibers. The fatigue behavior is mainly characterized by crack growth parameter $n_{d}$ (stress corrosion parameter). The value of $n_{d}$ for low-strength fibers which contain macroscopic flaws has not been sufficiently clarified, because only a few examinations have been made on low strength fibers $[2,3]$, although many studies have been made on high-strength fibers which have no macroscopic flaws $[4,5]$. The allowable loading condition needed to prevent any growth of the macroscopic flaw was discussed in order to assure high reliability for the fiber.

Chen and Chang [6] have focused on the fracture mechanics of silica optical fibers to evaluate the strength and fracture characteristics of single and multi-mode fibers subjected to uniaxial tensile testing and two-point bending. The fracture strength data of both single and multi-mode under either testing were found to be very similar. The fracture stresses at $50 \%$ fracture probability for tensile testing and two-point bending were 4.5 and $5.1 \mathrm{GPa}$, respectively. The fracture characteristics of each tested optical fiber specimen were evaluated by using an SEM. A critical flaw on the surface of glass fiber was found to be the fracture origin for specimens under either tension or bending as expected.

But, the influence of the coating on the fiber strength has not been plainly studied when the fiber is subjected to bending or tensile tests. The protective coating that surrounds the glass fiber plays an important role in protecting the glass cladding sur-face of the optical fiber from mechanical and chemical damages. However, the knowledge of the mechanical coating strength is one of the factors that determine the lifetime of the fabricated fiber-based devices. In this paper, silica optical fibers with and without polymer coatings were submitted to tensile and bending tests using a new stripping gel to remove the optical fiber coating materials that leaves the surface of the glass cladding intact.

\section{Fiber, stripping gel test benches used 2.1. Fiber used}

The used monomode silica fiber has an acrylate coating. This fiber was manufactured using the Plasma activated Chemical Vapor Deposition (PCVD) process which produces a totally synthetic, ultrapure fiber. The combined coating diameter is $242 \pm 5$ $\mu \mathrm{m}$, the clad diameter is $125 \pm 0.7 \mu \mathrm{m}$ and the coating thickness is $58.5 \pm 0.5 \mu \mathrm{m}$.

\subsection{Stripping gel used}

The used gel is a stripping gel based on methylene chloride and methanol. We have spread a gel layer on the surface of a glass plate specimen. Glass sample was exposed to gel during $20 \mathrm{~h}$ and cleaned using cellulose paper, rinsed with soap and ethanol. Figure 1 gives the surface details when AFM of microscope-grade was used for a glass surface not exposed to the stripping gel (Fig 1a) and that exposed to the stripping gel (Fig. 1b). One can note 
that there is no chemical reaction when stripping gel was spread. Gel used doesn't attack SiO2 molecules for a short term application such as coating stripping.

The surface roughness $S_{q}$ corresponds to the RMS (Root Mean Square gradient) of the altitude of the statistical distribution of heights in the sample.
$\mathrm{S}_{\mathrm{q}}$ varied from $0.64 \mathrm{up}$ to $2.20 \mathrm{~nm}$ for unexposed glass surface and from 0.7 up to $2.00 \mathrm{~nm}$ for exposed glass surface. Both measured $\mathrm{S}_{\mathrm{q}}$ correspond to the RMS of bulk microscope glass and indicate that there is no action between gel and $\mathrm{SiO} 2$ molecules during almost a day.

Figure 2 gives details of stripped fiber.
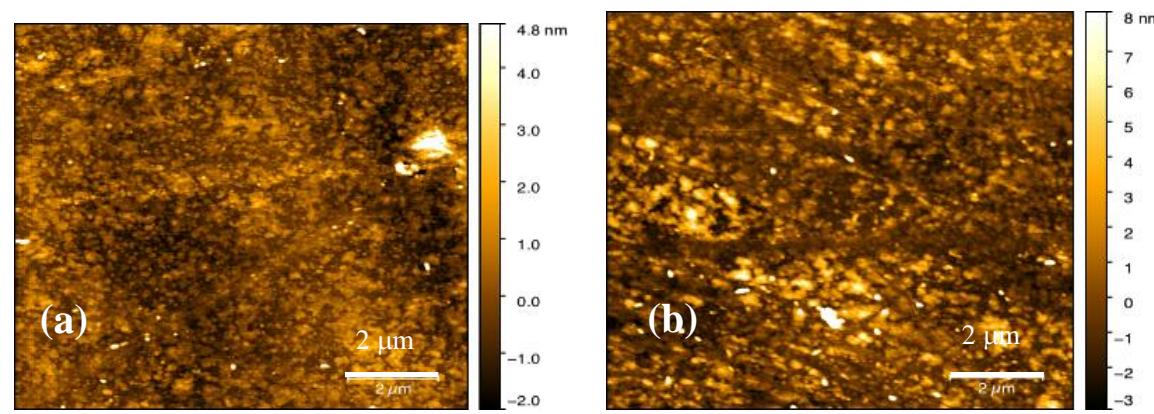

Fig. 1. (a) Glass surface without stripping gel and (b) exposed to stripping gel
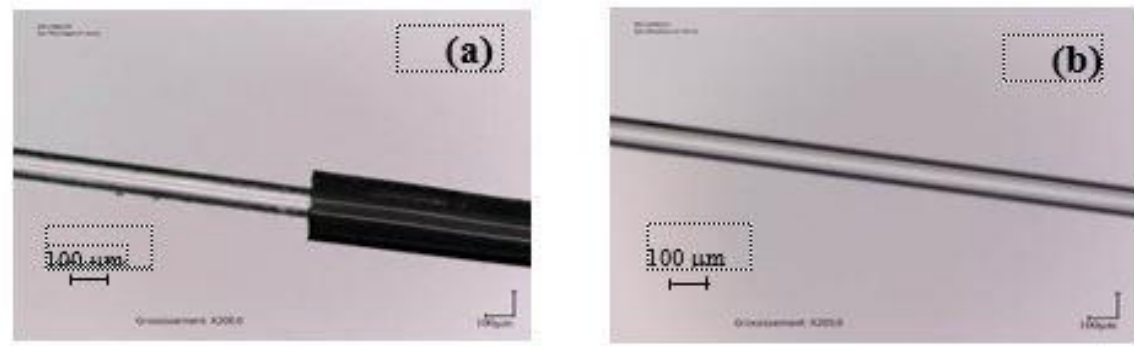

Fig. 2. Stripped optical fiber: (a) gel was applied only on a fiber part; (b) all fiber was stripped

\subsection{Test Benches Used}

Two experimental test benches were used:

a/. A two points bending bench made up of a displacement plate which is mounted on an aluminum plate. The first thrust block is movable and mounted on the displacement plate, while the second thrust block is fixed on a force sensor.
The optical fiber is positioned between the two thrust blocks in such a way that it forms a "U". To avoid slipping, the fiber is positioned in the grooves of the thrust blocks. During the test, load and displacement are recorded, allowing the load/displacement curve to be obtained (Fig. 3). Bending speeds were from 0.1 to $8.0 \mathrm{~mm} / \mathrm{s}$ and fiber length was $10 \mathrm{~cm}$.

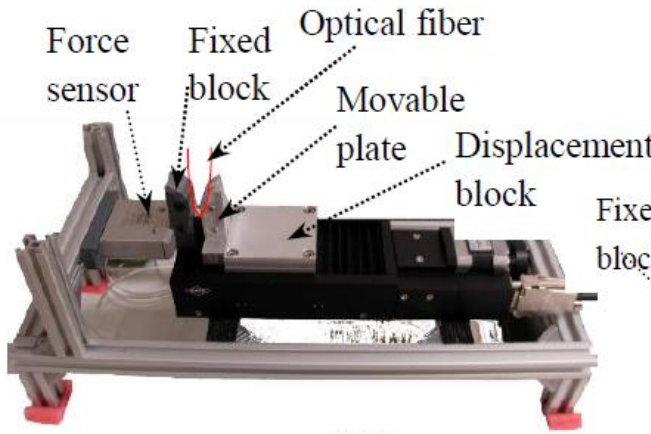

(a)

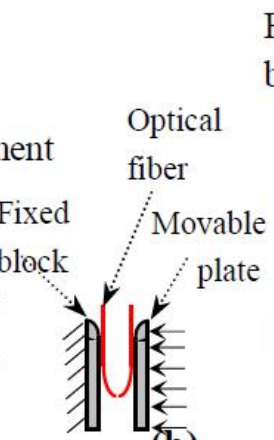

(b)

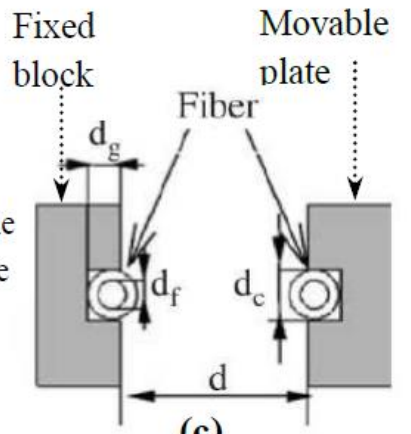

(c)

Fig. 3. (a) Bending bench used; (b) and (c) fiber between thrust blocks

b/. The dynamic tensile test consists of subjecting fibers to a deformation under a constant velocity until rupture. The used tensile bench was a Lloyd $50 \mathrm{~K}$ bench with a $100 \mathrm{~N}$ sensor (Fig. 4).

The fiber is rolled three times around two pulleys; the lower pulley is fixed and the upper pulley is movable with different velocities $(0.8,2.6$, $4.4,6.2$ and $8 \mathrm{~mm} / \mathrm{s}$ ).

These strain rates, expressed as a percentage of the initial sample length $(500 \mathrm{~mm})$, correspond to $1.610-3 \mathrm{~s}-1,5.2$ 10-3 s-1, 8.8 10-3 s-1, 12.4 10-3 s-1 and $1610-3 \mathrm{~s}-1$. 
Tensile testing was performed in a controlled environment with $46-52 \%$ relative humidity with a maximum of $5 \%$ humidity variation for each series of the tensile tests.

During the test, the tensile load was measured using a dynamometric cell (load sensor) while the fiber deformation was deduced from the displacement between the fixed lower pulley and the mobile higher pulley (Fig. 4). The testing procedure used 20 samples for each velocity.

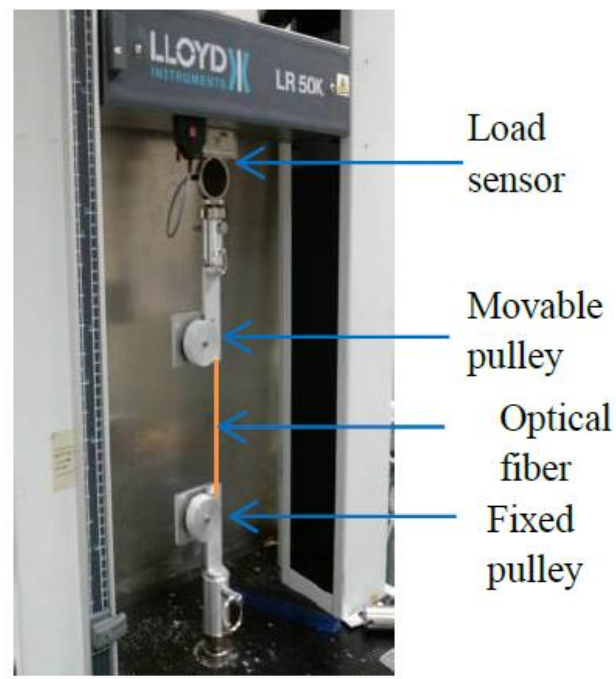

Fig. 4. Tensile test bench used

\section{Theoretical Approach}

The stress corrosion parameter $n_{d}$ refers to the crack growth parameter which is typically 20 or more for silica fiber.

The statistical Weibull law gives a relationship between the probability $\boldsymbol{F}$ of fiber rupture with a length $L$ and the applied stress $\sigma$.

$$
\operatorname{Ln}\left[\frac{1}{L}\left\{\operatorname{Ln}\left(\frac{1}{(1-F)}\right)\right\}\right]=m\left[\operatorname{Ln}(\sigma)-\operatorname{Ln}\left(\sigma_{o}\right)\right]
$$

where $m$ is a size parameter and $\sigma_{o}$ is a scale parameter.

The evolution of $\operatorname{Ln}\left[\frac{1}{L}\left\{\operatorname{Ln}\left(\frac{1}{(1-F)}\right)\right\}\right]$ (Failure cumulative probability in (\%) according to $\operatorname{Ln}\left(\sigma_{F}\right)$ is called Weibull diagram ( $\sigma_{F}$ is the failure stress). On the other hand, one can give the stress change according to the stress rate $\left[\dot{\sigma}_{F}\right]$ as follow:

$$
\operatorname{Ln}\left[\sigma_{F}\right]=\left(\frac{1}{1+n_{d}}\right) \operatorname{Ln}\left[\dot{\sigma}_{F}\right]+b
$$

The main parameter which defines the fiber mechanical strength was the stress corrosion parameter under dynamic loadings $n_{d}$.

Matthewson [7] gives for different applied strength the crack propagation type and the $n_{d}$ values (Table 1).

Table 1. Types of defect and strength ranges for silica [8]

\begin{tabular}{|l|c|c|l|}
\hline Defect Type & $\boldsymbol{n}_{\boldsymbol{d}}$ & Strength & \multicolumn{1}{|c|}{ Comments } \\
\hline Pristine & 20 & $>7 \mathrm{GPa}$ & Surface phenomena \\
\hline Subthreshold & $10-20$ & $0.3-7 \mathrm{GPa}$ & $\begin{array}{l}\text { Crack initiation dominates } \\
\text { by residual stresses }\end{array}$ \\
\hline Postthreshold & 30 & $1-300 \mathrm{GPa}$ & $\begin{array}{l}\text { Crack propagation } \\
\text { dominates by residual } \\
\text { stresses }\end{array}$ \\
\hline Iacroscopic crack & 40 & $<1 \mathrm{MPa}$ & $\begin{array}{l}\text { Sharp, residual stress free } \\
\text { cracks }\end{array}$ \\
\hline
\end{tabular}

\section{Results and Discussion}

Figures 5 and 6 give the Weibull distribution of coated and stripped fibers for tensile and bending tests for different faceplate velocities. For each velocity, the median fiber strengths of the coated or stripped fibers were close one to another. As a result, the presence of the coating modifies very little the mechanical strength of the fiber.

Table 2 gives $n_{d}$ values and the median strength for coated fibers and stripped fibers obtained from bending and tensile tests. The stress corrosion parameter $n_{d}$ is related to the Weibull curve slope.
This parameter varies little since all the failure stresses of stripped fiber have decreased by the same amount compared with those of the coated fiber.

If the coating provides protection to optical fiber against detrimental environmental effects and leads to a great fiber flexibility, it can also increase the fiber strength which is a significant parameter in the case of fibers used in telecommunication net-works. The strength loss for stripped fibers can be close to $10 \%$ when fiber is submit-ted to tensile test or to bending test. 

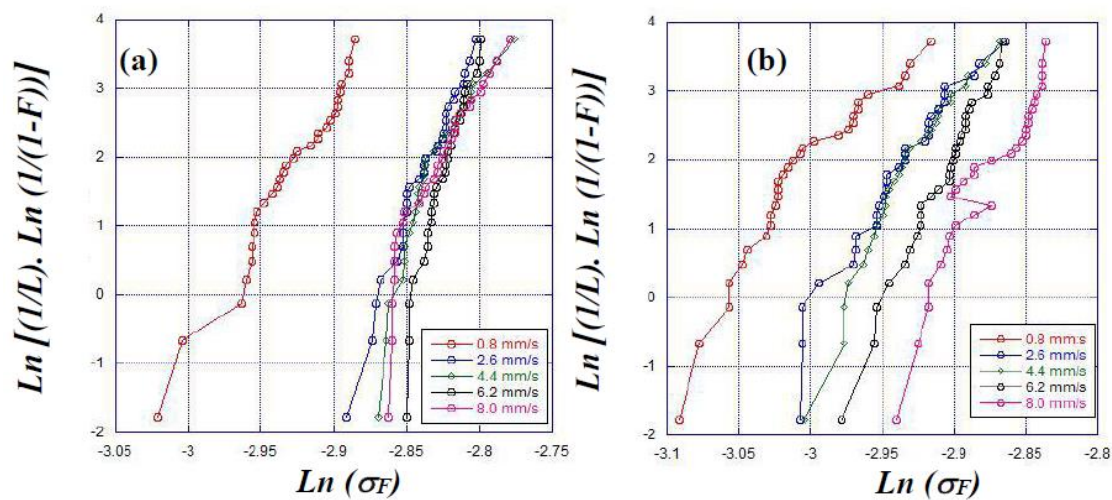

Fig. 5. Weibull curves for two point bending test: (a) coated fibers; (b) stripped fibers
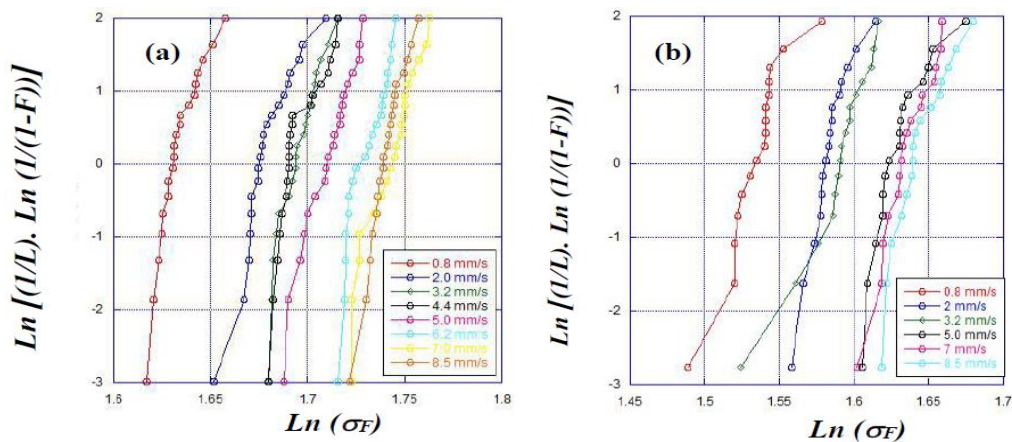

Fig. 6. Weibull curves for tensile test: (a) coated fibers; (b) stripped fibers

Table 2. Mechanical fiber parameters

\begin{tabular}{lcccc}
\hline & \multicolumn{2}{c}{ Coated fibers } & \multicolumn{2}{c}{ Stripped fibers } \\
\cline { 2 - 5 } & $\begin{array}{c}\text { Stress } \\
\text { corrosion } \\
\text { parameter } n_{d}\end{array}$ & $\begin{array}{c}\text { Median fiber } \\
\text { strength }(\mathrm{GPa})\end{array}$ & $\begin{array}{c}\text { Stress } \\
\text { corrosion } \\
\text { parameter } n_{d}\end{array}$ & $\begin{array}{c}\text { Median fiber } \\
\text { strength (GPa) }\end{array}$ \\
\hline Tensile test & 20.28 & $5.48 \pm 0.1$ & 18.61 & $4.96 \pm 0.13$ \\
\hline Bending test & 20.73 & $0.058 \pm 0.003$ & 21.72 & $0.052 \pm 0.003$ \\
\hline
\end{tabular}

\section{Conclusion}

A new tripping gel used allows for a clean and undamaged surface and AFM analysis confirms $\mathrm{SiO} 2$ isn't damaged on the short term. Stripping using gel allows for good measurements that were unreliable using other methods. In addition, only a few minutes after the coat of gel were sufficient to remove the fiber coating. Only 10\% mechanical resistance loss was due to coating stripping. The stress corrosion parameter nd changes little. The high value of $n_{d}$ obtained during the bending test for stripped fibers was certainly due to the difficulties during the experiment, because after the stripping of fibers, it is necessary to quickly test them otherwise they will be damaged by the environmental conditions (dust, oxygen, humidity ...).

\section{References}

[1] Sakaguchi, S., Hibino, Y.: Fatigue in low-strength silica optical fibres, J. of Materials Science 19, 3416-3420 (1984).
[2] Gulati, S. T., Helfinstine, J. D., Justice, B., McCartney, J. S. and. Runyan, M. A: Measurement of stress corrosion constant $\mathrm{n}$ for optical fibres, Amer. Ceram. Soc. Bull. 58, 1115-1117 (1979).

[3] Donaghy, F. A. and Nicol, D. R.: Evaluation of the fatigue constant $\mathrm{n}$ in optical fibers with surface particle damage, J. Amer. Ceram. Soc. 66, 601-604 (1983).

[4] Kalish, D. and Tariyal, B. K.: Static and dynamic fatigue of a polymer-coated fused silica optical fiber, J. Amer. Ceram. Soc. 61, 518-523 (1978).

[5] Sakaguchi, S. and Kimura, T.: Influence of temperature and humidity on dynamic fatigue of optical fibers, J. Amer. Ceram. Soc. 64, 259-262 (1981).

[6] Chandan, H. C. and Kalish, D.: Temperature dependence of static fatigue of optical fibers coated with a UV-curable polyurethane acrylate, J. Amer. Ceram. Soc. 65, 171-173 (1982).

[7] Matthewson, M. J.: Fiber optics reliability and testing, Optical fiber reliability models, SPIE Critical Reviews of Optical Science and Technology, Volume CR50, 3-31 (1993). 